\title{
A Very-Short-Term Intermittency of Fetal Heart Rates and Developmental Milestone
}

\author{
MYUNG-KUL YUM AND JONG-HWA KIM \\ Department of Pediatrics [M.-K.Y.], Hanyang University School of Medicine, Seoul Korea, 133-792; and \\ Department of Obstetrics \& Gynecology [J.-H.K.], School of Medicine Sungkyungkwan University, \\ Seoul, Korea, 135-701
}

\begin{abstract}
ABST
We noticed that fetal heart rates (FHR) of immature fetuses
intermittently showed unstable sudden and brief falls below
baseline FHR that occur over a few or several heartbeats. The
frightening falls do not occur in mature fetuses. In nonlinear
dynamics, the degree of such abrupt falls in time series is
quantified as intermittency. We aimed to investigate the nature
and maturational changes of intermittency of the FHR in normal
fetuses and to present the intermittency values of normal fetuses
according to gestational weeks. FHR data of 450 normal fetuses
between 23 and 40 wk of gestation were studied. We performed
multifractal analysis and highlighted a very-short-term intermit-
tency $\left(C_{1 \alpha}, 4 \leq\right.$ scale $<16$ heartbeats). The $C_{1 \alpha}$ values exhibited
a strong negative linear correlation $(r=-0.732, p=0.0001)$
with the gestational weeks. At $27-28,29-30,33-34$, and $37-38$
\end{abstract}
To overcome the recent challenge against usefulness of prenatal or intrapartum fetal heart rate (FHR) analysis $(1,2)$, we present a new FHR index whose values can estimate the degree of immaturity and the degree of unstable control of FHR. We noticed that immature fetuses intermittently showed severe sudden and brief falls in FHR below baseline FHR that occurs within a few or several successive heartbeats. Sudden falls of mild degree appeared more frequently. It was also observed that such sudden severe falls lessen as gestation advances. We also noticed that sick fetuses showed such unstable falls although they were fully matured. Therefore, we hypothesized that the degree of severe sudden falls could be a new FHR index that indicates the degree of immaturity. In the case of adequately matured fetuses, it indicates the degree of loss of normal mature heart rate control, i.e. the degree of cardiovascular sickness.

Received December 7, 2001; accepted September 16, 2002.

Correspondence: Prof. Myung-Kul Yum, Department of Pediatric Cardiology, Hanyang University Kuri Hospital, 249-1 Kyomun-Dong Kuri-Shi, Kyunggi-Do, 471-701, Korea; e-mail: mkyumm@hanyang.ac.kr

This work was supported by grant no. 1999-2-112-002-5 from the Interdisciplinary Research Program of KOSEF.

DOI: 10.1203/01.PDR.0000064945.53546.8E gestational weeks, the $C_{1 \alpha}$ values were significantly lower than those of the previous 2 or 4 gestational weeks. The maturation of normal fetuses is related to decreasing the severity of the unstable sudden and brief falls in FHR that is measured by $C_{1 \alpha}$, the very-short-term intermittency. The $C_{1 \alpha}$ values according to the gestational weeks that we presented can be used as credible normal milestone values when estimating the degree of maturity of certain FHR. Because the $C_{1 \alpha}$ captures the degree of the unstable fall in FHR, the $C_{1 \alpha}$ may also be used in identifying sick fetuses that are associated with hemodynamic instability and sudden bradycardia. (Pediatr Res 53: 915-919, 2003)

FHR, fetal heart rate

Abbreviations

Recent advances in nonlinear dynamics give us the opportunity to quantify various noise-like behaviors, such as sudden falls or jumps in time series, that are termed as intermittent behavior (3-11). The intermittent behavior is evaluated by multifractal analysis and quantified as intermittency.

In this article, we explore the nature of the intermittent behavior in FHR. Then a relation between the fetal maturation and degree of intermittency was analyzed to characterize the maturation of FHR dynamics in view of the intermittent behavior. In addition, the intermittency values according to the gestational weeks are presented and examined as to whether the values could be used as credible normal milestone values when estimating the degree of maturity of certain FHR.

\section{METHODS}

Selection of Subjects and Acquisition of FHR Data. After informed consent was obtained and the study was approved by the applicable Institutional Review Board, we analyzed the FHR of 450 normal fetuses that were associated with uncomplicated pregnancies and uneventful perinatal periods. The FHR data were recorded during the 23rd to 40th weeks of gestation. The data were recorded for $>40$ min with the mothers in a semirecumbent position using a Corometrics 115 (Corometrics, Minster, OH, U.S.A.) external monitor. The 
recorded data (FHR, uterine contraction, and fetal movements) were sampled into a personal computer with a digital serial interface. Whenever missing data were found, they were recorded as zero. The average signal loss in the analysis of the records was $2.4 \%$. When the off-line FHR data of 0 (missing data) or $<60$ beats per minute or $>200$ beats per minutes was encountered, they were removed. The 3000 points-FHR data where fetal movements were included-were used for calculating heart rate variability measures.

Power Spectral Analysis. For power spectral analysis of the heart rate data, the corresponding interheartbeat intervals were calculated. These were $1000 \mathrm{~Hz}$ linearly interpolated by their interheartbeat intervals to construct a real-time series of interheartbeat intervals and $2 \mathrm{~Hz}$ resampled. We extracted a 2048point $(2048 \div 60 \div 2 \mathrm{~Hz}=17.06 \mathrm{~min})$ time series of interheartbeat intervals. After removing a linear trend, the power spectral density function was estimated by the classical fast-Fourier transformation method. We calculated the lowfrequency and high-frequency components by integrating the power spectral density curve between the $0.04-0.15 \mathrm{~Hz}$ and the $0.15-0.5 \mathrm{~Hz}$ range, respectively (12).

Multifractal Analysis and Quantification of Intermittency. We denoted $t$-th heart rate as $\mathrm{y}(t)$. To quantify the intermittency in the FHR, we calculated the nonoverlapping fluctuations of difference in the heart rate $\Delta \mathrm{y}_{\tau}(t)=|\mathrm{y}(t+\tau)-\mathrm{y}(t)|$ for different time increment $\tau(3,9,10)$. To perform multifractal analysis, we estimated the statistical moments of these fluctuations, which depended on the time increment in a scaling way:

$$
<\left(\Delta y_{\tau}(t)\right)^{\mathrm{q}}>\sim \tau^{\mathrm{q}^{*} H(\mathrm{q})}
$$

To quantify the scaling behavior, the quantities, the $<\left(\Delta \mathrm{y}_{\tau}(t)\right)^{\mathrm{q}}>$ were plotted for $\log _{2}(\tau)=1 \ldots 10$ and $\mathrm{q}=0.1$, $0.4, \ldots, 2.8,3.1$. Find the scaling region and calculate the slopes

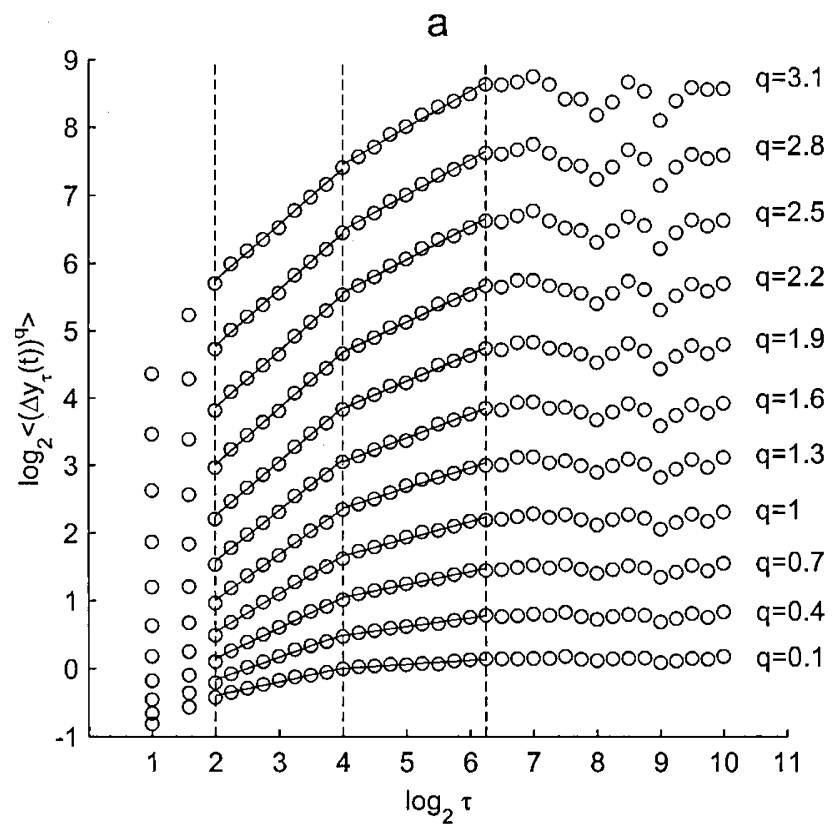

of the $\left\langle\Delta \mathrm{y}_{\tau}(t)^{q}\right\rangle$ against $\tau$ at the scaling region, which yield $\mathrm{q}^{*} H(\mathrm{q})$. The $H(\mathrm{q})$ is the generalized scale-invariant structure function exponent (Fig. 1a). The average of the fluctuations corresponds to $\mathrm{q}=1$, and the $H(1)$ is the parameter characterizing nonstationarity of the mean FHR.

The generalized intermittency exponent $C(\mathrm{q})$ is then calculated through the relation

$$
C(\mathrm{q})=-\mathrm{q}^{2} * \mathrm{~d} H(\mathrm{q}) / \mathrm{dq}
$$

Finally, the $C_{1}$ exponent, which is a measure of the average intermittency lying in the signal $\mathrm{y}(t)$, can be numerically estimated by measuring $C_{1}$ around $\mathrm{q}=1$ :

$$
C_{1}=-\mathrm{d} H(1) / \mathrm{dq}
$$

To calculate the value of $C_{1}$, a tangent line to $H(\mathrm{q})$ curve at the coordinate of $(1, H(1))$ was drawn (Fig. $1 b)$ and the slope of the tangent line was computed.

Statistical Methods. The mean value of $C_{1 \alpha}$ ranges from zero to unity. To test that the mean value at each of the 2-wk periods of gestational age is significantly larger than zero, we performed the signed rank test (SAS 8.01, SAS Institute, Cary, NC, U.S.A.). For linear correlation, the Pearson correlation coefficient was calculated. Kruskal-Wallis test was used to assess significant differences in each heart rate index between the gestational weeks, with Mann-Whitney rank-sum test to test the significance of the differences.

\section{RESULTS}

When we plotted the values of $\log _{2}<\left(\Delta \mathrm{y}_{\tau}(t)\right)^{\mathrm{q}}>$ versus $\log _{2 \tau}$, at $\tau<4$ heartbeats or $\tau>80$ heartbeats, there were no consistent linear scaling relationships (Fig. 1). However, at $4 \leq$ $\tau \leq 80$ heartbeats, two distinct linear scaling relations could be

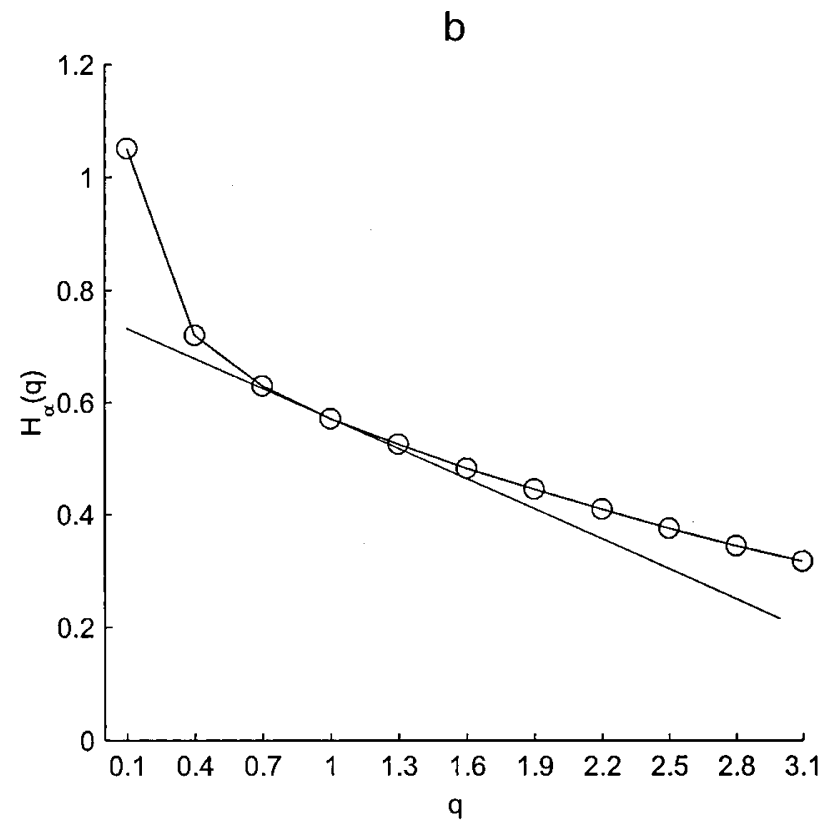

Figure 1. $a$, Multifractal analysis and the scaling behavior of the quantities $\left\langle\Delta y \tau(t)^{q}\right\rangle$ : we have indicated two linear scaling ranges, the very-short-term range ( $4 \leq \tau<16$ heartbeats) and the short-term range ( $16 \leq \tau \leq 80$ heartbeats), and the slopes of the $<\Delta \mathrm{y} \tau(t)^{q}>$ against $\tau$ at the scaling ranges are the $\mathrm{q}^{*} H \alpha(\mathrm{q})$ and q $^{*} H \beta(\mathrm{q})$, respectively. $b$, A plot of $H \alpha(\mathrm{q})$ against q. Notice a tangent line that is drawn to calculate $C_{1 \alpha}$ (see "Methods"). 
seen and the separations of the linear scaling behavior happened at approximately $\tau \cong 16$ heartbeats. Therefore, we could obtain very-short- and short-term generalized structure function exponents $(H \alpha(\mathrm{q}), 4 \leq \tau<16$ heartbeats, and $H \beta(\mathrm{q}), 16$ $\leq \tau \leq 80$ heartbeats, respectively) from equation 1 and corresponding very-short- and short-term generalized intermittency exponents $(C \alpha(\mathrm{q}), 4 \leq \tau<16$ heartbeats, and $C \beta(\mathrm{q}), 16$ $\leq \tau \leq 80$ heartbeats, respectively) from equation 2 . For $\mathrm{q}=1$, we obtained average very-short-term and short-term intermittency exponents $\left(C_{1 \alpha}, C_{1 \beta}\right.$, respectively) from equation 3 (see "Methods").

In general, $C$ quantifies the degree of the singularity-a behavior that markedly deviates from the usual and expected one from the aspect of dynamical property $(3,6,7,9,10)$. Because the scale of the $C_{1 \alpha}$ ranged from four to 15 heartbeats, it quantifies the very-short-lasting singularity in the FHR. Therefore, the $C_{1 \alpha}$ can quantify the behavior of sudden falls in the heart rates frequently encountered in immature fetuses. Likewise, because the scale of the $C_{1 \beta}$ ranged from 16 to 80 heartbeats, the $C_{1 \beta}$ quantifies the degree of the singularity that lasts longer than 16 heartbeats in the same FHR. In this study, we considered only $C_{1 \alpha}$.

The conventional statistical indices mean and SD showed significant but weak linear correlations (Table 1). The spectral indices high- and low-frequency power did not show significant linear correlations. The $C_{1 \alpha}$ values exhibited a strong negative linear correlation $(r=-0.732, p=0.0001)$ with the gestational weeks (Table 1 ). The $C_{1 \beta}$ values also showed a significant negative linear correlation $(r=-0.422, p=$ 0.0001 ) with the gestational weeks. The 2 -wk period averaged values of the $C_{1 \alpha}$ and $H_{1 \alpha}$ are illustrated in Table 2. The average values of $C_{1 \alpha}$ at all gestational weeks were significantly $(p<0.001)$ larger than zero. The average value of $C_{1 \alpha}$ at 23-24 wk was the highest among all gestational ages and thereafter decreased consistently as gestation advanced. It decreased significantly as early as at 27-28 wk compared with that of 23-24 wk $(p<0.005)$. Further significant decreases occurred at 29-30 $(p<0.01), 33-34(p=0.0001)$, and 37-38 $(p=0.0001)$ weeks of gestation.

Three representative FHR time series of 23, 31, and $40 \mathrm{wk}$ of gestation are illustrated in Figure 2, left. On visual inspection of that of the 23rd week (Fig. $2 a$, left); there are intermittent episodes of sudden falling of the heart rate below the baseline of the heart rate time series. The arrow in Figure $2 a$ indicates a representative episode whose zoomed in version is shown in Figure $1 a$, right. FHR falls rapidly within four heartbeats from 152 beats per minute down to 128 beats per

Table 1. Correlation analysis of heart rate indices with gestational weeks $(n=450)$

\begin{tabular}{lc}
\hline \multicolumn{1}{c}{ FHR indices } & $\begin{array}{c}\text { Correlation coefficient } \\
(p)\end{array}$ \\
\hline Mean heart rate & $-0.246(0.0005)$ \\
SD of heart rate & $0.287(0.0001)$ \\
High-frequency power & $0.039(0.551)$ \\
Low-frequency power & $0.116(0.081)$ \\
Very-short-term intermittency $\left(C_{1 \alpha}\right)$ & $-0.7328(0.0001)$ \\
Hurst exponent $\left(H_{1 \alpha}\right)$ & $0.230(0.0001)$ \\
\hline
\end{tabular}

minute. Similar rapid falls are also found in the heart rate time series of the 30th week of gestation (Fig. $2 b$, left). However, the degree of the fall is less than that of the $23 \mathrm{rd}$ week of gestation (Fig. $2 b$, right). In contrast, the mature term fetus of 40th week of gestation rarely show the significant fall, which is below the baseline of its heart rate time series (Fig. $2 c$, left, solid arrow), and, if present, the degree of the fall is much smaller and very gradual over $>10$ heartbeat sequences (Fig. $2 c$, right). The $C_{1 \alpha} \mathrm{s}$, the degree of such sudden and brief falls, of the three fetuses are $0.145,0.079$, and 0.019 , respectively, and can differentiate the three fetuses quantitatively.

\section{DISCUSSION}

In this article, we introduced the so-called intermittent behavior to unveil a new pattern of FHR dynamics. Because of the unique nature of the FHR dynamics, we were able to find that the two different intermittent behaviors could coexist in an FHR. Consequently, we termed them as very-short- and shortterm intermittent behavior, which were quantified $C_{1 \alpha}$ and $C_{1 \beta}$, respectively. With the $C_{1 \alpha}$, we were able to quantify the degree of the sudden and brief falls in heart rates, frequently occurring in immature fetuses.

We demonstrated that, unlike the statistical and spectral indices, the $C_{1 \alpha}$ decreased in a strongly linear manner as the gestational weeks increased. Furthermore, at 23-24 wk of gestation, the $C_{1 \alpha}$ was the highest among all of the gestational periods. The first significant decrease compared with the $C_{1 \alpha}$ at 23-24 wk occurred at 27-28 wk, and at 29-30, 33-34, and 37-38 wk the significant decrease compared with the previous 2-4 weeks continues. At term gestation, although the $C_{1 \alpha}$ was not equal to zero, it decreased to approximately one ninth of that figure at 23-24 wk of gestation. Therefore, $C_{1 \alpha}$ values of FHR can credibly specify the gestational weeks that the $C_{1 \alpha}$ values according to gestational weeks can be a reliable normal developmental milestone of FHR after 23rd week.

From the significant and continuous decrease with advancing gestation, it is revealed that fetal maturation is strongly related to decreasing the $C_{1 \alpha}$ value, the degree of sudden and brief falls in FHR. The contractile apparatus in the fetus's myocardium is limited in increasing or decreasing stroke volume (13). Consequently, the cardiac outputs largely depend on the heart rate. For this reason, the presence of severe sudden and brief falls in the heart rate may suddenly decrease the cardiac output and be harmful. Therefore, suppressing the degree of the risky and immature very-short-term intermittent behavior with advancing gestation must be a natural and purposive maturational process.

In this study, we did not study the mechanism of the suppression with advancing gestation. The significant decrease in $C_{1 \alpha}$ compared with that at 23-24 wk occurred at 27-28 wk, when the cardiac innervations of the sympathetic (14) and parasympathetic nerve (15) and development of baroreceptor (16) for short-term cardiovascular control occur. Therefore, the appearance and maturation of autonomic nervous system and baroreceptor without a doubt contribute to the suppression of risky falls in FHR. 
Table 2. $C_{1 \alpha}$ and $H_{1 \alpha}$ values of the FHR according to gestational weeks

\begin{tabular}{lccccc}
\hline & \multicolumn{3}{c}{$C_{1 \alpha}$} & & \multicolumn{2}{c}{$H_{1 \alpha}$} \\
\cline { 2 - 4 } Gestational weeks & Median & $5 \mathrm{p}$ & $95 \mathrm{p}$ & Median & $5 \mathrm{p}$ \\
\hline $23-24(n=20)$ & 0.130 & 0.094 & 0.176 & 0.473 & 0.369 \\
$25-26(n=30)$ & 0.115 & 0.0625 & 0.145 & 0.479 & 0.334 \\
$27-28(n=40)$ & 0.100 & 0.051 & $0.146^{*}$ & 0.503 & 0.411 \\
$29-30(n=60)$ & 0.076 & 0.039 & $0.112 \dagger$ & 0.523 & 0.402 \\
$31-32(n=60)$ & 0.063 & 0.007 & 0.105 & 0.514 & 0.563 \\
$33-34(n=60)$ & 0.043 & 0.006 & $0.089 \dagger$ & 0.482 & 0.406 \\
$35-36(n=60)$ & 0.034 & -0.028 & 0.085 & 0.519 & 0.341 \\
$37-38(n=60)$ & 0.030 & -0.015 & $0.074 \S$ & 0.503 & 0.333 \\
$39-40(n=60)$ & 0.023 & -0.08 & 0.069 & 0.519 & 0.395 \\
\hline
\end{tabular}

$5 \mathrm{p}, 5$ th percentile; $95 \mathrm{p}, 95$ th percentile.

$* P<0.005$ compared with $\leq 24$ wk.

$\dagger P<0.01$ compared with $\leq 28$ wk.

$\ddagger P=0.0001$ compared with $\leq 30$ wk.

$\S P<0.0001$ compared with $\leq 34 \mathrm{wk}$

बा $P<0.01$ compared with $\leq 26$ wk.
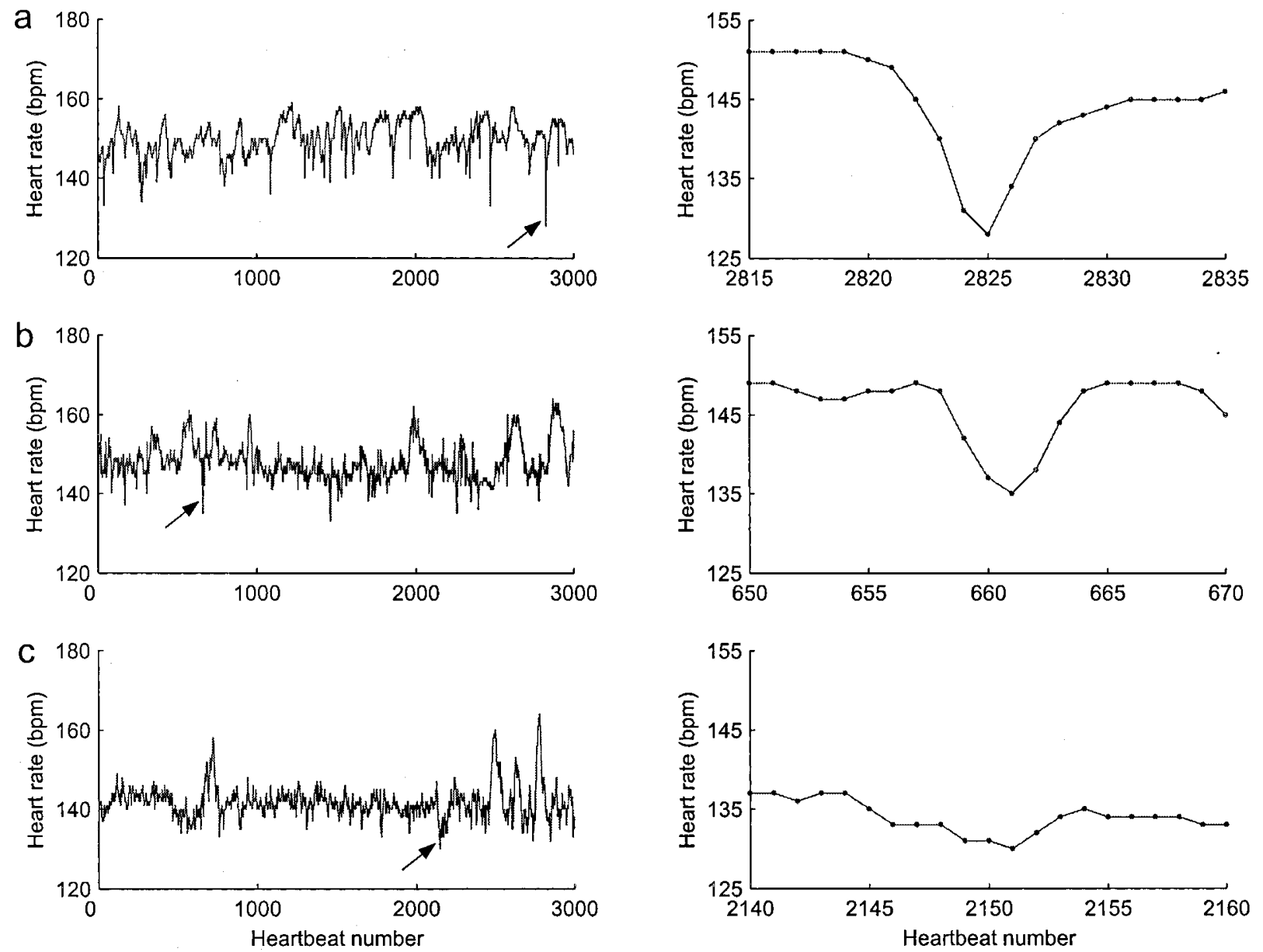

Figure 2. Heart rate time series of the fetuses aged 23 ( $a$, left), 31 ( $b$, left), and 40 ( $c$, left) weeks and their zoom versions (right). The arrows indicate the very-short-term intermittent behaviors (see "Results").

We have shown that the $C_{1 \alpha}$ can be a milestone for maturation of heart rate dynamics. More important, it can be an indicator of the cardiovascular sickness itself. When $C_{1 \alpha}$ value of a fetus at a certain gestational week exceeds the milestone value of that week, it indicates that the fetus's heart rate tends to fall more suddenly and deeply, the fetus's heart rate control is unstable, and the fetus's cardiovascular system is sick. Therefore, the $C_{1 \alpha}$ may be used for identifying sick fetuses who are associated with hemodynamic instability and sudden bradycardia. We succeeded in that the $C_{1 \alpha}$ can identify fetuses who will show late deceleration during labor and low 1-min Apgar score after birth among fetuses from mothers with pregnancy-induced hypertension (data not shown). 
In addition to the $C_{1 \alpha}$, the $C_{1 \beta}$ significantly decreased with advancing gestation. This result indicates that the decrease in the short-term intermittency that lasts for $>16$ heartbeats is another characteristic of the maturational process. However, because the correlation coefficient of $C_{1 \beta}$ was not high, $C_{1 \beta}$ cannot be used as a credible normal milestone value.

As discussed hitherto, the multifractal analysis was useful in quantifying average intermittency in the FHR, which revealed a new maturational characteristic in FHR. The usefulness of the multifractal analysis on heart rate was also exemplified by a study of Ivanov et al. (3). They performed the multifractal analysis on Wavelet Transform Maximum Modulus in human heart rate to calculate local Hurst exponent. Using this local Hurst exponents and Legendre transformation, they could highlight the nature of local correlation or anticorrelation of heart rates: diversity (multifractality) and distribution (fractal dimension) of the local correlation.

In conclusion, we presented the new heart rate index of FHR, the very-short-term intermittency, which captured the degree of the unstable sudden and brief fall in FHR. With the help of the index, it was unveiled that fetal maturation is strongly associated with suppressing unstable sudden falls in FHR. Its values according to the gestational weeks presented here can be used as credible normal milestone values when estimating the degree of maturity of certain FHR. Because it is unique in capturing unstable sudden falls in FHR, it will be useful for identifying sick fetuses who are associated with hemodynamic instability and sudden bradycardia.

\section{REFERENCES}

1. Parer JT, King T 2001 Fetal heart rate monitoring: is it salvageable? Am J Obstet Gynecol 182:982-987

2. Gagnon R, Bocking AD, Richardson BS, McLean PA 1994 Flat fetal heart rate tracing with normal fetal heart rate variability. Am J Obstet Gynecol 171:1379-1381

3. Ivanov P, Amaral LAN, Goldberger AL, Havlin S, Rosenblum MG, Struzik Z, Stanley HE 1999 Multifractality in human heartbeat dynamics. Nature 399:461-465

4. Dunki RM, Keller E, Meier PF, Ambuhl B 2000 Temporal patterns of human behaviour: are there signs of deterministic 1/f scaling? Physica A 276:596-609

5. Rodelsperger F, Cenys A, Benner H 1995 On-off intermittency in spin-wave instabilities. Phys Rev Lett 75:2594-2597

6. Bickel DR 2002 Generalized entropy and multifractality of time series: relationship between order and intermittency. Chaos Solitons Fractals 13:491-497

7. Latora VV, Belkacem M, Bonacera A 1994 Dynamics of instabilities and intermittency. Phys Rev Lett 73:1765-1768

8. Bickel DR 1999 Simple estimation of intermittency in multifractal stochastic processes: biomedical applications. Phys Lett A 262:251-256

9. Riedi RH, Crouse MS, Ribeiro VJ, Baraniuk RG 1999 A multifractal wavelet model with application to network traffic. IEEE Transact Inform Theory 45:992-1018

10. Davis A, Marshak A, Wiscombe W, Cahalan R 1994 Multifractal characterizations of nonstationarity and intermittency in geophysical fields: observed, retrieved, or simulated. J Geophys Res 99:8055-8072

11. Vandelwalle N, Ausloos M 1998 Sparseness and roughness of foreign exchange rates. Int J Mod Phys C 9:711-719

12. Akselrod S, Gordon D, Ubel FA, Shannon DC, Barger AC, Cohen RJ 1981 Power spectrum analysis of heart rate fluctuation: a quantitative probe of beat to beat cardiovascular control. Science 213:220-222

13. Grant DA, Fauchere JC, Eede KJ, Tyberg JV, Walker AM 2001 Left ventricular stroke volume in the fetal sheep is limited by extracardiac constraint and arterial pressure. J Physiol 535:231-239

14. Barret CT, Heymann MA, Rudolph AM 1972 Alpha and beta adrenergic function in fetal sheep. Am J Obstet Gynecol 112:1114-1121

15. Vapaavouri EK, Shinebourne EA, Williams RL, Heymann MA, Rodolph AM 1973 Development of cardiovascular responses to autonomic blockade in intact fetal and neonatal lambs. Biol Neonate 22:177-188

16. Shinebourne EA, Vapaavuori EK, Williams RL 1972 Development of baroreflex activity in unanesthetized fetal and neonatal lambs. Circ Res 31:710-718 